\title{
Uncertainty assessment approach for composite structures based on global sensitivity indices
}

\author{
C.A. Conceição António, L.N. Hoffbauer
}

\begin{abstract}

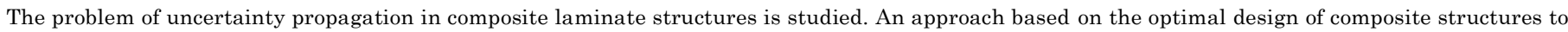

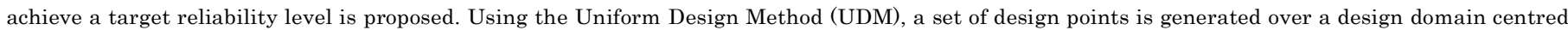

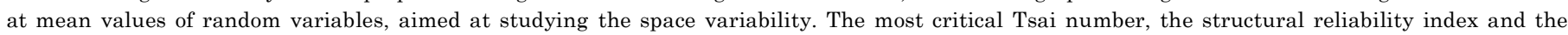

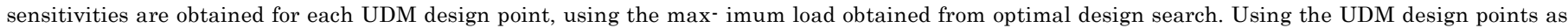

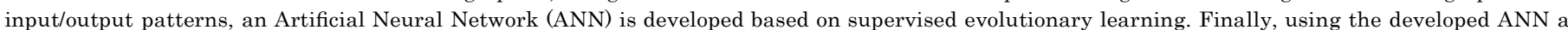

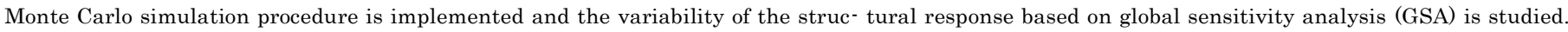

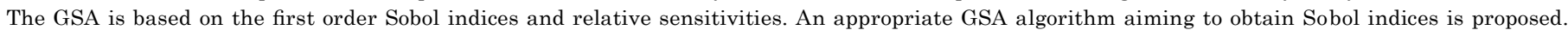
The most important sources of uncertainty are identified.
\end{abstract}

Keywords

Uncertainty Reliability Composites Global sensitivity Sobol indices ANN-MCS

\section{Introduction}

Composite materials behavior is extremely affected by numerous uncertainties that should be considered in structural design. The problem of design-based uncertainty of laminated composite structures can be formulated as an optimization problem or addressed as the problem of alleviating the effects of unavoidable parameter uncertainties. The first perspective is associated to reliability-based design optimization (RBDO) and the second one is considered in robust design optimization (RDO). Both strategies depend on uncertainty propagation analysis of composite structures response and different length scales.

Nowadays the definition of structural design criteria is based on ultimate state theory rather than on service stress theory. The application of such concepts to composite materials based on reliability analysis creates new challenges to the designer. A comprehensive review paper on RBDO developments is presented by Frangopol and Maute [1]. Recent works in RBDO applied to composite structures have been presented. Rais-Rohani and Singh [2] discuss the development of global and sequential response surface techniques for reliability-based optimization of composite structures under axial compression and buckling instability. Singh et al. [3] investigated the influence of variations of material prop- erties on the elastic stability of laminated composite panels. Adali et al. [4] developed a model for the optimal design of composite laminates under buckling load uncertainty.

The structural tailoring technique was applied to design laminated composite structures by searching the stacking sequence that corresponds to the less sensitive performance properties relatively to uncertainties in the input parameters. This perspective follows RDO concepts where the objective is to minimize the effects of uncertainty on optimal design. The strategy is based on considering the statistical data in objective and constraint functions [5].

Although several methods have been presented for uncertainty assessment, their efficiency was not proven, in particular when applied to composite structures [6,7]. The almost totality of sensitivity analyses in applications with composite structures used local importance measures of design parameters [2-10]. In particular Rais-Rohani and Singh [2] and Carbillet et al. [9] studied the sensitivity of reliability index of composite structures with non-linear behavior and quantified the importance of the random variables using local measures. Although the innovative aspects of joint reliability and sensitivity analysis, the use of local importance measures of uncertainty propagation is limited. So, Global Sensitivity Analysis (GSA) on the uncertainty response is still unexplored, remaining an open issue.

The uncertainty propagation of composite structures is investigated in this work considering descriptive statistical measures of the response variability and sensitivity analysis of system 

responses inside GSA framework [11-13]. A study based on sensitivity to uncertainty that allows selecting the important parameters using global sensitivity indices is presented. The uncertainty propagation and the importance measure of input parameters are analyzed using an Artificial Neural Network-based Monte Carlo simulation approach (ANN-MCS). The proposed methodology uses a Monte Carlo procedure together an Artificial Neural Network surrogate model based on supervised evolutionary learning [14].

The use of approximate models in reliability analysis has been studied. In particular, ANN has been used to approximate the limit state function and its derivatives proposed a hybrid technique based on ANN in combination with genetic algorithms (GA) for structural reliability analysis [15-17]. Following a different procedure, an approach based on an ANN model simulating at the same time the limit state function, the reliability index and their sensitivities is proposed in this paper. The objective is to study the propagation of uncertainties of mechanical properties on the response of composite laminate structures (linear mechanical behavior) under an imposed reliability level. Robustness assessment of the reliability-based designed composite structures is considered and some criteria are outlined for the particular case of angle-ply laminates. The longitudinal elastic mod-

ulus $E_{1}$, transversal elastic modulus $E_{2}$, transversal strength in tensile $Y$, and shear strength $S$ are considered the ANN input variables. These are the mechanical properties with the most critical deviations on the composite laminate strength randomness, according to the numerical simulation performed by Conceição António [18] and António et al. [19]. Nevertheless, the presented study can be extended to other random variables.

The paper is organized as follows: Section 2 presents the formulation of the uncertainty propagation analysis describing the main features of the ANN-MCSproposed approach, theinversereliability analysis and the ANN developments. GSA proposed model is described in Section 3. The numerical applications are presented in Section 4 together with the discussion of the results. Finally the conclusions on the performance of the proposed approach are presented in Section 5.

\section{Uncertainty propagation analysis}

\subsection{ANN-MC approach}

The objective of the proposed approach is to study the propagation of uncertainties in input random variables, such as mechanical properties, on the response of composite laminate structures for a specified reliability level. Fig. 1 shows the proposed Artificial Neural Network based Monte Carlo simulation procedure. The proposed approach for uncertainty propagation analysis in RBDO of composite structures for the particular case of angle-ply laminates is addressed according to the following steps:

1st Step: An approach based on optimal design of composite structures to achieve a specified reliability level, $\beta_{Q}$, is considered, and the corresponding maximum load is calculated as a function of ply angle, $a$. This inverse reliability problem is solved for the mean reference values, $\bar{\pi}_{4}$, of mechanical properties of the composite laminates.

2nd Step: Using the Uniform Design Method, a set of design points belonging to the interval $\left[\bar{\pi}_{i}-\alpha \bar{\pi}_{i}, \bar{\pi}_{i}+\alpha \bar{\pi}_{i}\right]$ is gener ated. covering a domain centered at mean reference values of the random variables. This method enables a uniform exploration of the domain values necessary in the development of an ANN approximation model for variability study of the reliability index.

3rd Step: For each UDM design point, the most critical Tsai number, $\bar{R}$, assodiated with the most probable failure point (MPP), structural reliability index, $\beta_{\mathrm{s}}$, and their relative sensitivity indices, $S_{\mathrm{m}}$, are obtained using the previously calculated maximum load for mean values, $\tilde{\pi}_{i}$, as a reference. The Hasofer-Lind method is used for reliability index assessment $[18,20,21]$. The sensitivity analysis is performed by the adjoint variable method $[18,20]$.

4th Step: An ANN is developed based on supervised evolutionary learning. The generated UDM design points and their calculated response values are used as input/output patterns. 5th Step: Using the developed ANN-based Monte Carlo simulation procedure, the variability of the structural reliability index and the critical Tsai number are evaluated as a function of ply angle domain. The uncertainty propagation is studied using the first order Sobol indices and relative sensitivities.

\section{Inverse reliability analysis}

For a target reliability index $\beta_{a}$, the inverse problem can formulated as follows:

$$
\begin{array}{ll}
\text { Minimize } & {\left[\beta_{s}\left(\lambda, a, \mu_{s}\right)-\beta_{a}\right]^{2}} \\
\text { Subject to } & 0 \leqslant a \leqslant \frac{\pi}{2}
\end{array}
$$

where $\beta_{s}$, is the structural reliability index, $\mu_{\pi}$ is the realization of random variable $\pi$. The mean values, $\bar{\pi}_{\text {, }}$ of mechanical properties of composite laminates are considered for $\mu_{\pi}$. The design variables are the ply angle, $a$, and load factor, $\lambda$.

The vector of applied loads is defined as $\mathbf{L}=\lambda \mathbf{L}^{\text {ref }}$, where $\mathbf{L}^{\text {eff }}$ is the reference load vector and after solution of the problem in Eq (1) the corresponding maximum load is computed for each value of ply angle $a$. This is a conventional RBDO inverse optimization problem. To solve the inverse problem (1), a decomposition of the problem is considered. The Lind-Hasofer method and appropriate iterative scheme based on a gradient method are applied to evaluate the structural reliability index, $\beta_{5}$, in the inner loop $[18,20,21]$. From the operational point of view, the reliability problem can be formulated as the constrained optimization problem

$$
\begin{array}{ll}
\text { Minimize } & \beta(\mathbf{v})=\left(\mathbf{v}^{T} \mathbf{v}\right)^{1 / 2} \\
\text { Subject to } & \varphi(\mathbf{v})=0
\end{array}
$$

where $\mathbf{v}$ is the vector of the standard normal variables, $\beta$ is the reliability index and $\varphi(\mathbf{v})$ is the limit state function. The relationship between the standard normal variables and random variables is established using the following projection formula:

$v_{1}=\frac{\pi_{1}-\bar{\pi}_{1}}{\sigma_{\pi_{i}}}$

where $\bar{\pi}_{i}$ and $\sigma_{\mathrm{s}_{i}}$ are, respectively, the mean values and standard deviations of the basic random variables. The limit state function that separates the design space into failure $(\varphi(\pi)<0)$ and safe regions $(\varphi(\pi)>0)$ can be written as

$\varphi(\pi)=R-1$

where $\bar{R}$ is the critical Tsai number, defined as

$\bar{R}=\operatorname{Min}\left(R_{1}, \ldots, R_{k}, \ldots, R_{N_{s}}\right)$

and $N_{s}$ the total number of points where the stress vector is evaluated. The Tsai number, $R_{k}$, which is a strength/stress ratio [22], is obtained from the Tsai-Wu interactive quadratic failure criterion and calculated at the $k$ th point of the structure solving equation

$1-\left(F_{i j} s_{i} s_{j}\right) R_{k}^{2}+\left(F_{i} s_{i}\right) R_{k}=0$

where $s_{i}$ are the components of the stress vector, and $F_{i j}$ and $F_{i}$ are the strength parameters associated with unidirectional reinforced 


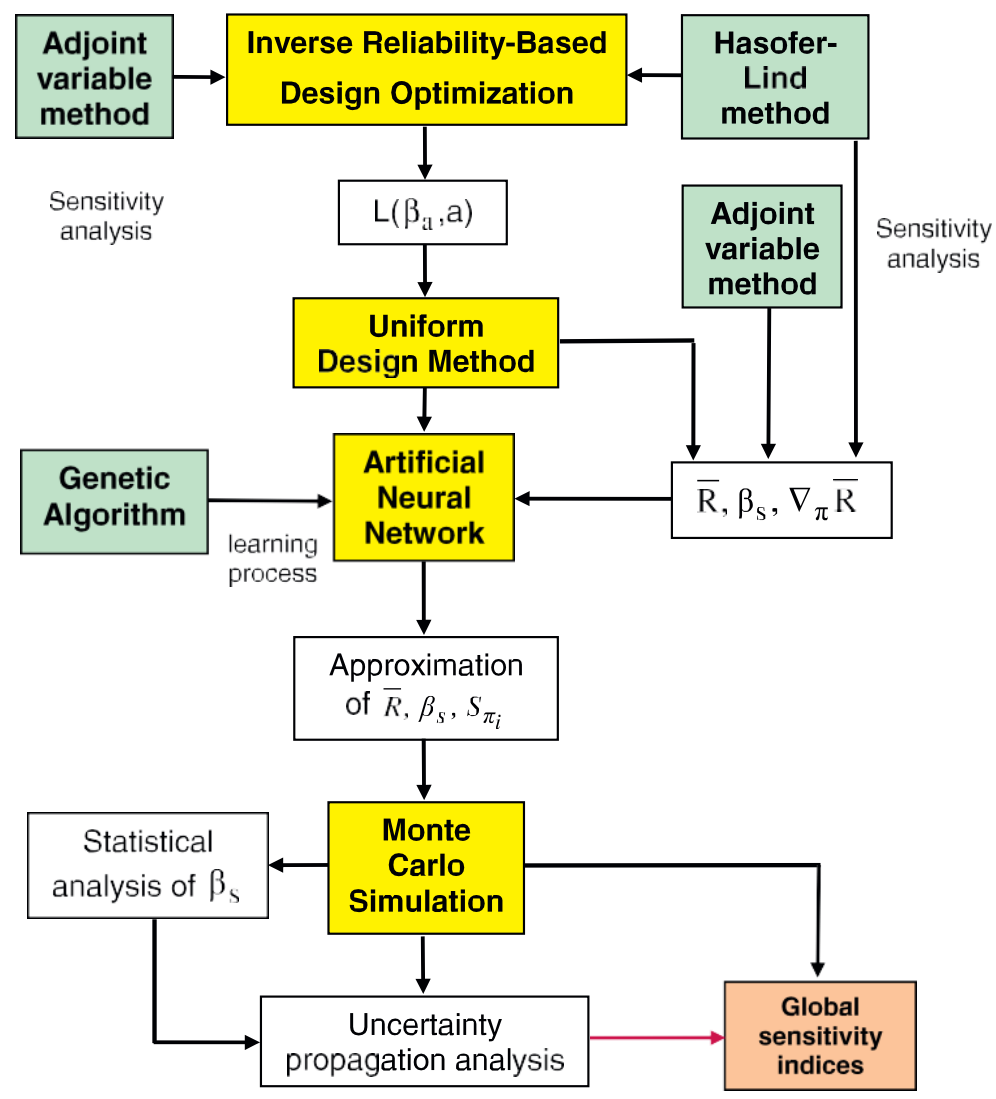

Fig. 1. Flowchart of proposed approach for uncertainty propagation analysis.

laminate defined from the macro-mechanical point of view [22]. The solution, $v^{*}$, of the reliability problem in Eq. (2) is referred to, in technical literature, as the design point or most probable failure point (MPP). The bisection method used to estimate the load factor, $k$, is iteratively used in the external loop [23]. After the minimization of the objective function given in Eq. (1), the structural reliability index is $b_{s} \curvearrowright b_{a}$ with some prescribed error, and the corresponding load vector is $\mathrm{L}\left(b_{a}\right)$.

\subsection{ANN developments}

The proposed ANN is organized into three layers of nodes (neurons): input, hidden and output layers. The linkages between input and hidden nodes and between hidden and output nodes are denoted by synapses. These are weighted connections that establish the relationship between input data and output data.

In the developed ANN, the input data vector $\mathrm{D}^{\text {inp }}$ is defined by a set of values for random variables $p$, which are the mechanical properties of composite laminates, such as elastic or strength properties. Following the developments performed by Conceição António [18] and António et al. [19] only the critical mechanical properties of composite laminates are selected as ANN input data. The objective is to avoid exhaustive calculations with high computational costs. Using a modified version of the Monte Carlo analysis the referred authors $[18,19]$ proposed a methodology based on a parametric study of the influence of the physical properties randomness in angle-ply laminated composite strength and furthermore the choice of the most relevant mechanical properties. The parametric study concluded that the most important properties for angle-ply laminates randomness strength are the longitudinal elastic modulus $E_{1}$, transversal elastic modulus $E_{2}$, transversal strength in tensile $Y$, and shear strength $S$. So, this mechanical properties are considered as ANN input variables and denoted by $\mathrm{p}=\left[E_{1}, E_{2}, Y, S\right]$. Nevertheless, the presented study can be extended to other random variables.

In the proposed ANN-MC approach, each set of input values for the random variable vector $\mathrm{p}$ is selected using the Uniform Design Method (UDM) [24]. The procedure is based on a UDM table denoted by $U_{n}\left(q^{s}\right)$, where $\mathrm{U}$ is the uniform design, $\mathrm{n}$ the number of samples, q the number of levels of each input variable, and $s$ the maximum number of columns of the table. For each UDM table, there is a corresponding accessory table, which includes a recommendation of columns with minimum discrepancy for a given number of input variables. Using the UDM a set of design points belonging to the interval $\left.1 / 2 p_{i}-a p_{i} ; p_{i} \mathrm{p} a p_{i}\right]$ is generated, covering a domain centered at mean reference values of the random variables. This method enables a uniform exploration of the domain values necessary in the development of an ANN approximation model guarantying better results after learning procedure [25]. The corresponding output data vector $\mathrm{D}^{\text {out }}$ contains the critical Tsai number, $\bar{R}$, structural reliability index, $b_{s}$, and relative sensitivities $S_{p_{i}}$ of reliability index with respect to random variables. The concept of relative sensitivity [26] of the reliability index is defined as

$$
S_{\pi_{4}}=\left|\frac{\partial \beta_{s}}{\partial \pi_{i}}\right| \frac{\pi_{1}}{\beta_{s}} \mid
$$

and its analysis aims to compare the relative importance of input parameters on the response. Fig. 2 shows the topology of the ANN, showing the input and output parameters.

Each pattern, consisting of an input and output vector, needs to be normalized to avoid numerical error propagation during the ANN learning process [25]. The activation of the $k$ th node of the hidden layer $(p=1)$ and output layer $(p=2)$ is obtained through sigmoid functions. The error between predefined output data and 


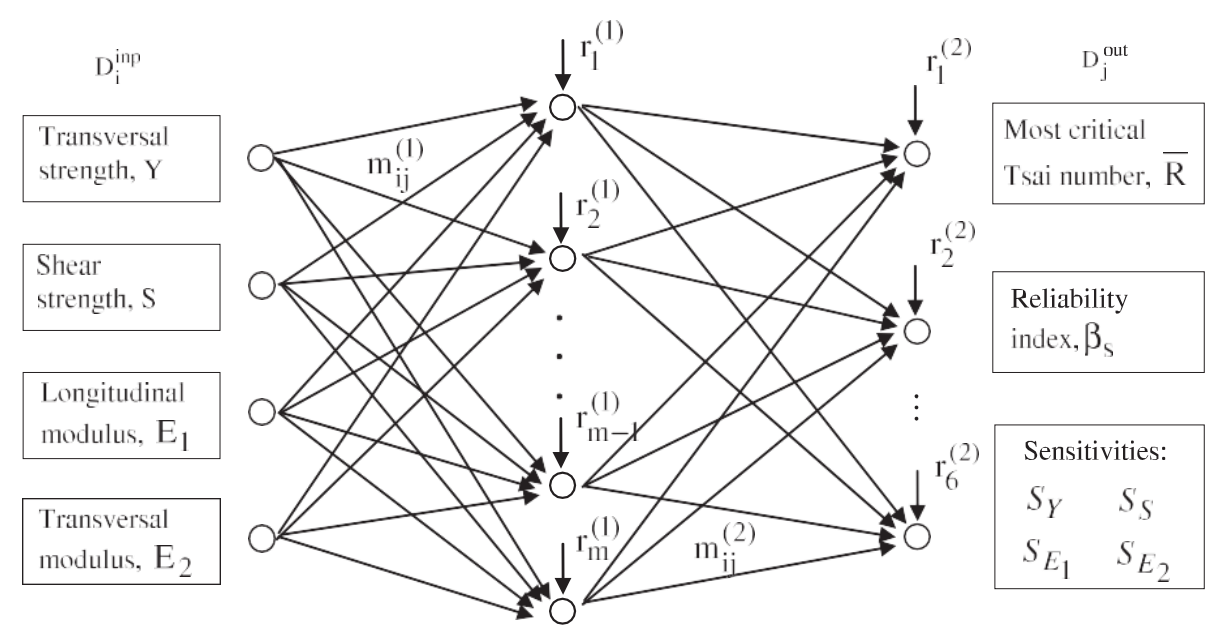

Fig. 2. Artificial Neural Network topology.

ANN simulated results is used to supervise the learning process, which is aimed at obtaining a complete model of the process. As a set of input data are introduced to the ANN, it adapts the weights of the synapses and values of the biases to produce consistent simulated results thropugh a process known as learning. The weights of the synapsess, $\mathrm{m}_{i j}$, and biases in the neurons at the hidden and output layers, ${ }_{k}$, are controlled during the learning process. For each set of input data and any configuration of the weight matrix and biases, a set of output results is obtained. These simulated output results are compared with the predefined values to evaluate the difference (error), which is then minimized during the learning procedure.

The adopted supervised learning process of the ANN based on a Genetic Algorithm (GA) [27] uses the weights of synapses and biases of neural nodes at the hidden and output layers as design variables. A binary code format is used for these variables. The number of digits of each variable can be different depending on the connection between the input-hidden layers or hidden-output layers. A GA is an optimization technique based on the survival of the fittest and natural selection theory proposed by Charles Darwin. The genetic algorithm [27] basically performs on three parts: (1) coding and decoding random variables into strings; (2) evaluating the fitness of each solution string; and (3) applying genetic operators to generate the next generation of solution strings in a new population. Three basic genetic operators, namely selection, crossover, and mutation are used in this paper. An elitist strategy based on conservation of the best-fit transfers the best-fitted solution into a new population for the next generation. Once the new population is created, the search process performed by the three genetic operators is repeated and the process continues until the average fitness of the elite group of the current generation no longer shows significant improvement over the previous generation. Further details on creating and using a genetic algorithm for ANN learning can be found in the reference [27].

\section{Global sensitivity analysis}

The local measures of sensitivity are not enough for a full evaluation of the influence of input parameters on structural response uncertainty [12-14]. The uncertainty analysis on response in the neighborhood of mean values of input parameters is of limited value. To obtain the influence of individual parameters on the uncertainty at the output structural response $W_{m}$ Global Sensitivity Analysis (GSA) techniques must be used. Global Sensitivity Analysis denotes the set of methods that consider the whole variation range of inputs and tries to share the output response uncertainty among the input parameters.

\subsection{Global variance-based method}

Among GSA techniques the variance-based methods are the most appropriate $[12,13,28]$. GSA studies the effects of input variations on model outputs in the entire allowable ranges of the input space. Global Sensitivity Analysis (GSA) has an advantage over lo-

cal sensitivity analysis in that GSA does not require strong model assumptions such as linearity or monotonicity [13,28]. However its application for composite structures is complex and expensive from the computational point of view. In this work the variancebased methods is applied to a group of input parameters namely the physical properties of composites and then compared with local importance measures.

Assuming that $\mathbf{X}=\left(X_{1}, \ldots, X_{n}\right)$ are $n$ independent input parameters and $\Psi_{m}$ is the performance function of structural response previously defined, an indicator of the importance of an input parameter $X_{i}$ is the following normalized index

$S_{l}=\frac{\operatorname{var}\left(E\left\langle\Psi_{m} \mid X_{l}\right\rangle\right)}{\operatorname{var}\left(\Psi_{m}\right)}$

named first-ordersensitivity index proposed by Sobol $[11,13,28,29]$. In Eq. (8) $\operatorname{var}\left(E\left\langle\Psi_{m 1} \mid X_{i}\right\rangle\right)$ is the variance of the conditional expectation and $\operatorname{var}\left(\Psi_{m}\right)$ is the variance of $\Psi_{m}$. Furthermore, Sobol $[11,13,28,29]$ proposed a complete variance decomposition of the uncertainty associated with $\psi_{m}$ into components depending on individual parameters and interactions between individual parameters. This procedure explains the variance $\operatorname{var}\left(\Psi_{m}\right)$ as a contribution of the partial variance associated to each individual parameter $[11,13,28,29]$. From this decomposition higher order sensitivity indices can be established in particular the second order sensitivity index. The second order index $S_{y}$ defines the sensitivity of the structural response $\Psi_{m}$ to the interaction between $X_{i}$ and $X_{\text {, i.e. the portion of the }}$ variance of $\Psi_{m}$ that is not included in the individual effects of $X_{i}$ and $X$. The sum of all order indices is equal to 1 in case all input parameters are independent $[11,28,29]$. Since higher order sensitivity indices require tedious calculations only the Sobol first-order sensitivity index is used in the presented work $[11,14,29]$.

\subsection{GSA evaluation using Monte Carlo simulation}

One of the problems using global sensitivity indices is the computational cost. Due to the large number of input parameters in the uncertainty propagation analysis on composite structures, Finite Element Method evaluations become very expensive. In this work the ANN-based Monte Carlo simulation approach is used for the estimation of GSA indices. To reduce the computational costs the 
analysis is implemented using groups of input parameters and considering only the Sobol first-order sensitivity index. [14]:

The proposed methodology is based on the following algorithm

1st Step: Lets consider $p$ groups of non-correlated input parameters $\pi=\left(\pi_{1}, \ldots, \pi_{p}\right)$ following a normal distribution $N$ with mean $\bar{\pi}_{i}$ and standard deviation $\sigma_{i}$ represented by $\pi_{i} \sim N\left(\bar{\pi}_{i}, \sigma_{i}\right)$. 2nd Step: Considers a set of random numbers, $\Gamma_{f=x}=\left(\lambda_{4}, \ldots, \lambda_{s},\right)$, following a standard normal distribution $N(0,1)$. These random numbers are used to generate the fixed values of the input parameter $\pi_{i}$ :

3rd Step: For each input parameter $\pi_{j \neq i}$ a sample matrix is generated by independently collecting samples of $(p-1)$ random numbers following a normal distribution $N(0$,$) :$

$$
\mathbf{M}_{\alpha}=\left[\begin{array}{ccc}
\alpha_{1,1} & \ldots & \alpha_{1 p-1} \\
\vdots & \ddots & \vdots \\
\alpha_{N_{r}, 1} & \cdots & \alpha_{N_{r, p-1}}
\end{array}\right]
$$

where the size of the sample is $N_{r}$.

\section{4th Step:}

Repeat for each input parameter $\pi_{i, i}=1 \rightarrow p$

Do $k=1 \rightarrow N_{f}$

Do $q=1 \rightarrow N_{r}$

$$
\pi_{j}^{k^{k}}=\left\{\begin{array}{ll}
\pi_{j}+\lambda_{k} \sigma_{j} & \text { if } j=i \\
\pi_{j}+\alpha_{q j} \sigma_{j} & \text { if } j \neq i
\end{array} \quad \text { for } j=1, \ldots, p\right.
$$

Evaluation of the structural response: $\Psi_{m}\left(\pi^{k, 9}\right)$, the vector $\pi^{k, 9}$ being the nominal values of $\pi$, with components $\pi_{j}^{k, 4}$.

\section{End Do}

Estimate the conditional expectation of structural response function $\Psi_{m}$ by:

$$
E\left(\Psi_{m} \mid \pi_{i}\right) \approx \Psi_{m}^{k}=\frac{1}{N_{r}} \sum_{q=1}^{N_{r}} \Psi_{m}\left(\pi^{k, q}\right)
$$

\section{End Do}

Estimate of the mean values

$$
\overline{\bar{\Psi}}_{m}=\frac{1}{N_{f}} \sum_{k=1}^{N_{f}} \bar{\Psi}_{m}^{k}
$$

Estimation of the variance of the conditional expectation of structural response, fixing the input parameter $\pi_{i}$ :

$$
\left.\operatorname{var}\left(E\left\langle\Psi_{m}\right| \pi_{i}\right)\right) \approx \frac{1}{N_{f}-1} \sum_{k=1}^{N_{f}}\left(\bar{\Psi}_{m}^{k}-\overline{\bar{\Psi}}_{m}\right)^{2}
$$

\section{End repeat}

5th Step: Estimation of variance of structural response var $\left(\Psi_{m}\right)$ considering the previous $N_{T}=N_{r} \times N_{f} \times p$ simulations for $\Psi_{m}$ :

$$
\begin{aligned}
& E\left(\Psi_{m}\right)=\frac{1}{N_{T}} \sum_{i=1}^{p} \sum_{k=1}^{N_{f}} \sum_{q=1}^{N_{f}}\left[\Psi_{m}\left(\pi^{k, q}\right)\right]_{i} \\
& \operatorname{var}\left(\Psi_{m}\right)=\frac{1}{N_{T}-1} \sum_{i=1}^{p} \sum_{k=1}^{N_{f}} \sum_{q=1}^{N_{\tau}}\left\{\left[\Psi_{m}\left(\pi^{k q}\right)\right]_{i}-E\left(\Psi_{m}\right)\right\}^{2}
\end{aligned}
$$

6th Step: Calculation of the global sensitivity index:

$$
S_{l}=\frac{\operatorname{var}\left(E\left(\Psi_{m} \mid \pi_{l}\right)\right)}{\operatorname{var}\left(\Psi_{m}\right)} \quad i=1, \ldots, p
$$

\section{Numerical examples}

\subsection{Clamped cylindrical shell}

To test the proposed approach applied to composite structures, a clamped cylindrical shell laminated structure is considered, as shown in Fig. 3. Nine vertical loads with mean value $L_{k}$ are applied along the free linear side $(A B)$ of the structure. This free linear side $(A B)$ is constrained in the $y$-axis direction. The structure is made of one laminate. The balanced angle-ply laminates with eight layers and stacking sequence $[-a /+a /-a /+a]_{s}$ are considered in a symmetric construction. Ply angle, $a$, is referenced to the $x$-axis of the reference coordinate, as detailed in Fig. 3. All plies have a thicknesses of $2.5 \times 10^{-3} \mathrm{~m}$.

The structural analysis of laminated composite structures is based on the finite element method (FEM) and shell finite element model developed by Ahmad [30], and indudes improvements from Figueiras [31]. The Ahmad shell element is obtained from a 3-D finite element using a degenerative procedure. It is an isoparametric element with eight nodes and five degrees of freedom per node, as described by Mindlin shell theory.

The laminate is made of a carbon/epoxy composite system [22]. The mean reference values of the elastic and strength properties of the ply material used in the laminate construction of the composite structure are presented in Table 1 . The elastic constants of the orthotropic ply are the longitudinal elastic modulus, $E_{1}$; transversal elastic modulus, $E_{2}$; in-plane shear modulus, $G_{12}$; out-of-plane shear modulus, $G_{13}$ and $G_{23}$; and in-plane Poisson's ratio, $v_{12}$. The ply strength properties are the longitudinal strength in tensile, $X$; longitudinal strength in compression, $X$; transversal strength in tensile, $Y$; transversal strength in compression, $Y$; and shear strength, $S$.

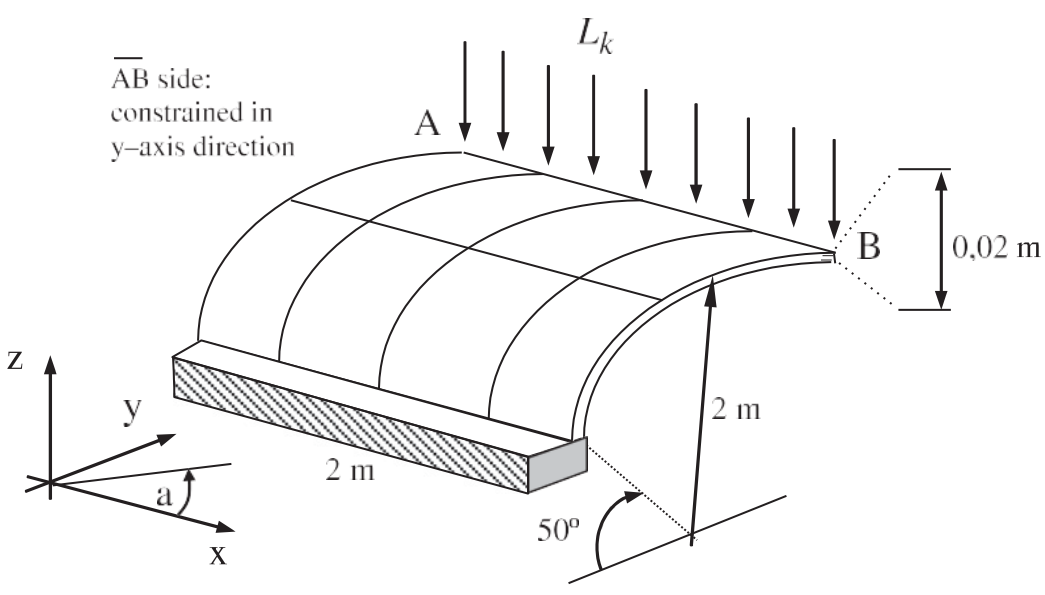

Fig. 3. Geometric and loading definition of cylindrical composite shell 
Table 1

Mean reference values of mechanical properties of composite layers.

\begin{tabular}{|c|c|c|c|c|c|c|c|c|}
\hline Material & $E_{1}(\mathrm{GPa})$ & $E_{2}(\mathrm{GPa})$ & $\mathrm{G}_{12}(\mathrm{GPa})$ & $m$ & $X ; X^{0}(\mathrm{MPa})$ & $Y ; Y^{0}(\mathrm{MPa})$ & $S(\mathrm{MPa})$ & $q\left(\mathrm{~kg} / \mathrm{m}^{3}\right)$ \\
\hline T300/N5208 & 181.0 & 10.3 & 7.17 & 0.28 & $1500 ; 1500$ & $40 ; 246$ & 68 & 1600 \\
\hline
\end{tabular}

To assess reliability, the longitudinal elastic modulus, $E_{1}$, trans versal elastic modulus, $E_{2}$, transversal strength in tensile, $Y$, and shear strength, $\mathrm{S}$, are the considered random variables and denoted by $\mathrm{p}=\left[E_{1}, E_{2}, Y, S\right]$. All random variables are non-correlated, and follow a normal probability distribution function defined by their respective mean and standard deviation. The present study can be further extended to other random variables. To obtain the maximum reference load, the inverse RBDO problem defined in Eq. (1) is solved. The structural reliability index is $b_{s} \geqslant b_{a}$ with some pre scribed error, and the corresponding maximum load vector, $\mathrm{L}\left(b_{a}\right)$, can be obtained. The reliability assessment follows the procedure described in Eqs. (2)-(6). A target reliability index $b_{a}=3$ for the composite structure is considered. The mean values of the mechanical properties are assumed to be random variables and are defined in Table 1, and the coefficient of variation of each random variable is set to $\mathrm{CV}(\mathrm{p})=6 \%$, relative to the mean value.

The Most Probable failure Point (MPP) values are obtained based on the Hasofer- Lind method. After obtaining these values, the inverse RBDO, formulated in Eq. (1), is solved for $b_{a}=3$ and the maximum load is outlined depending on ply angle, $a$. The corresponding maximum load is plotted as a function of ply angle, $a$, and shown in Fig. 4. This load is used as the reference load for further uncertainty propagation analysis in the ANN-MCS and supported by UDM and GA developments.

The UDM points are considered as experimental input values to be used in the ANN learning procedure. A number of 27 training data sets is selected inside the interval $k p_{i}-0: 06 p_{i} ; p_{i}$ p 0:06 $\left.p_{i}\right]$, with mean reference value $p_{i}$ set as a random variable for each mechanical property and defined in Table 1.

In Uniform Design Method (UDM) originally proposed by Fang et al. [24] a set of design points is generated over a domain centered at mean values of random variables, aimed at studying the space variability. Obtaining points that are most uniformly scattered in the s-dimensional unit cube $C^{s}$ is the key of UDM, which

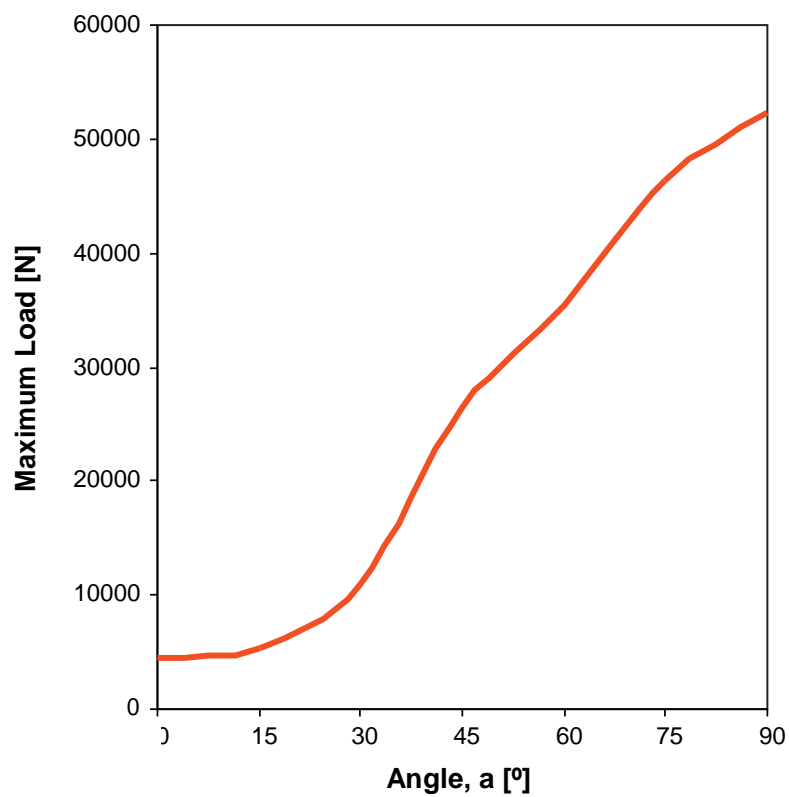

Fig. 4. Maximum load for $b_{a}=3$, solving the inverse RBDO problem for clamped composite shell. is based on a quasi-Monte Carlo method. In this context, the discrepancy is used as a measure of uniformity that is universally accepted. As referred in Section 2.3, for each UDM table there is a corresponding accessory table, which includes a recommendation of columns with minimum discrepancy for a given number of input variables.

The UDM values are selected according to the approach proposed by Cheng et al. [16]. After selecting Table $U_{27}\left(27^{10}\right)$ of the UDM [16], where columns 1, 4, 6 and 9 must be selected according to the respective accessory Table for four variables and discrepancy $W(n, P)=0.1189$, the resulting integer code format is presented in Table 2. The UDM table must be transformed into a hyper-rectangle region corresponding to the input variable domain by linear transformation. Then the interval $1 / 2$ p $p_{i} \quad 0: 06 p_{i} ; p \mathrm{p} \quad 0: 06 p$ ] is equally discretized with 27 points and, using the integer code for mat from Table 2, the actual composition for $\mathrm{p}=\left[E_{1}, E_{2}, Y, S\right]$ is obtained [25].

Reliability analysis is performed for the input values from $\mathrm{Ta}^{-}$ ble 2 and 27 input/output patterns are obtained and used in ANN development. For each UDM design point, the most critical Tsai number, $R$, associated with the most probable failure point (MPP); reliability index of structure, $b_{s}$; and relative sensitivities are obtained by using the maximum load previously calculated for each ply angle, a considering the respective domain, as a reference and solving the inverse RBDO formulation of Eq. (1). A fixed standard deviation $r_{p_{i}}{ }^{1} \mathbb{Q}: 06 p_{i}$ is used in the reliability index evaluation for all UDM design points, based on Hasofer-Lind method. The sensitivities are calculated based on the adjoint variable method $[18,20]$.

A number of 10 neurons are considered for the hidden layer of the ANN topology. The ANN learning process is formulated as an optimization problem with 116 design variables corresponding to

Table 2

UDM design points for discrepancy $W(n, P)=0.1189$

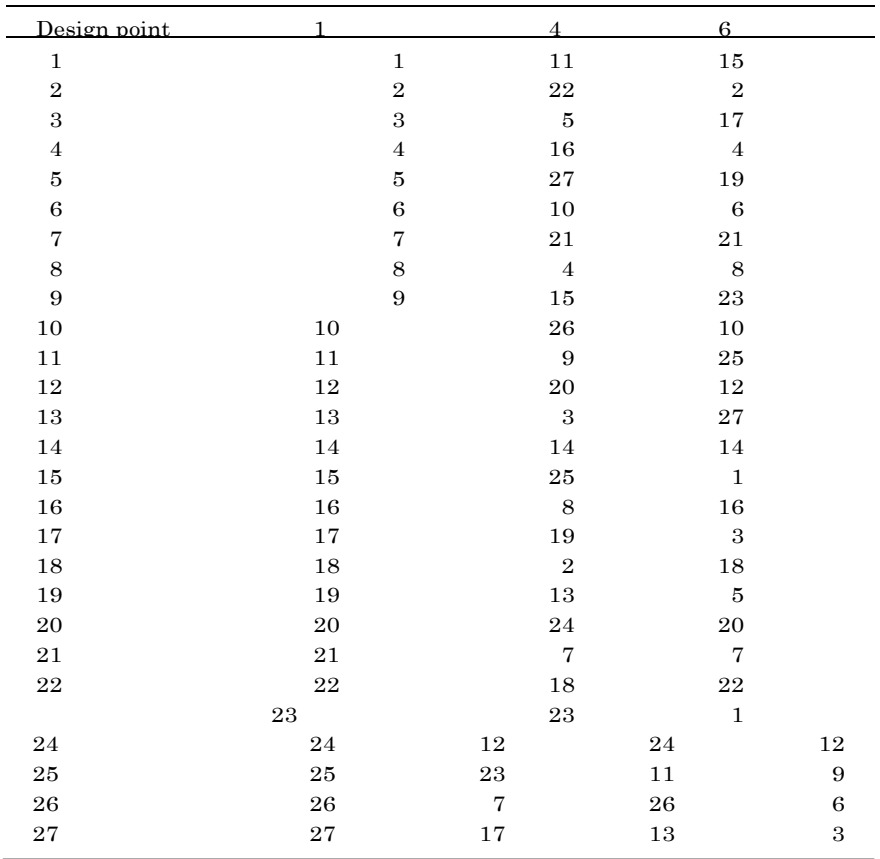


100 weights of synapses and 16 biases of neural nodes [25]. The ANN-based GA learning process is performed using a population of 21 individuals/solutions. The elite and mutation groups have 7 and 4 solutions, respectively [27]. The binary code format with 5 digits is adopted for both designing the values of the weights of synapses and biases of neural nodes at the hidden and output layers. The learning process is concluded after 15,000 generations of the GA. The mean values in Table 1 (point 14 of UDM Table 2) are used for ANN testing. The relative errors in learning and testing processes corresponding to the optimal ANN are less than $1 \%$.

Using the proposed ANN-MCS approach 5000 simulations are obtained aiming to analyze the behavior of structural response parameters as the critical Tsai number, the reliability index and its relative sensitivities. An example of the implemented analysis is shown in Fig. 5 with the relative sensitivities of the reliability index $b_{s}$ calculated for ply angle $a=60^{\circ}$. The histograms show that the response do not follow a Normal probability distribution function.

Descriptive statistics of the structural response parameters calculated for ply angle $a=60^{\circ}$ are presented in Table 3. Supported by the statistical analysis it can be concluded that all response parameters, except the critical Tsai number, show large variations. The coefficients of variation (C.V.) are very high when compared to the coefficient of variation for the input random variables, which has a predefined value of $6 \%$ of the mean values. This is confirmed also by box plot analysis in Fig. 6 .

The importance of input parameters on uncertainty propagation on structural response is shown in Figs. 6 and 7 based on box-plot analysis. In particular, the variability of the reliability index in RBDO and associated relative sensitivities indices must be considered for robust design of composite structures.
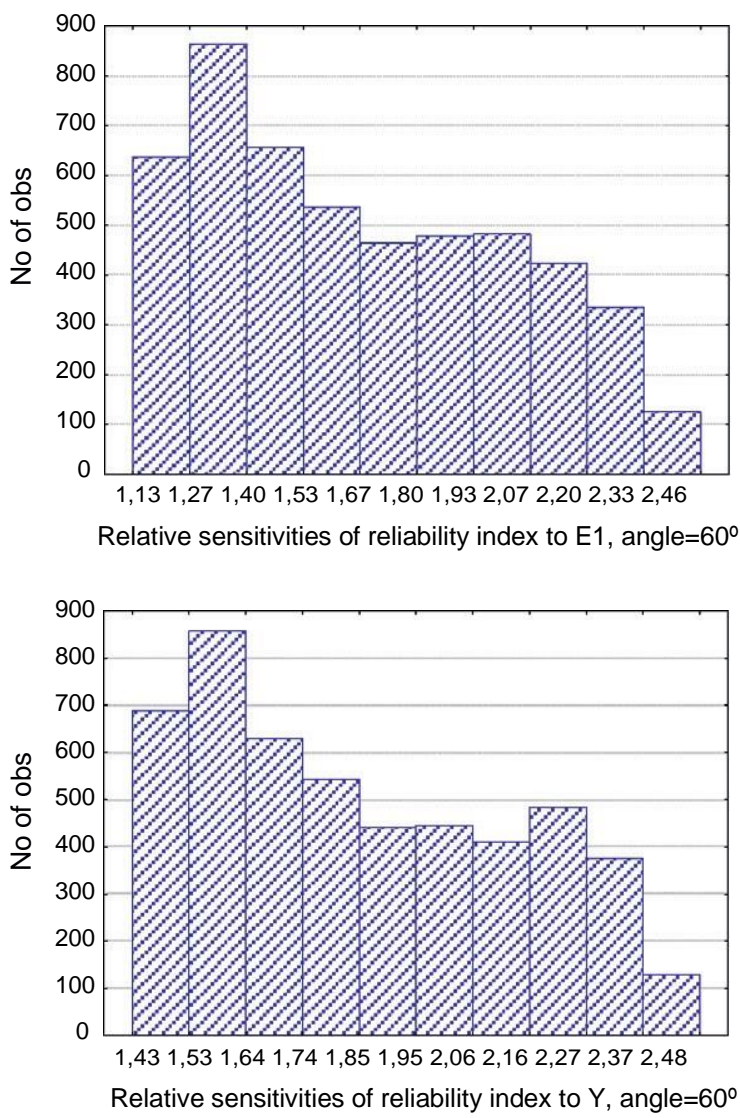

Fig. 7 shows the interval of variation for the relative sensitivities obtained from Eq. (7). The objective is to compare the relative importance of the input parameters on structural response, in particular for the inverse RBDO solutions. The reliability index $b_{s}$, is very sensitive to transversal strength, $Y$, over the entire domain of angle $a$ except for $a=45^{\circ}$. The sensitivity relative to the shear strength, $S$, is high for ply angle values equal to $45^{\circ}$ and $60^{\circ}$. The relative sensitivities of other parameters as longitudinal elastic modulus and transversal elastic modulus are not so important.

The global variance-based method proposed in Section 3.2 and ANN-based Monte Carlo simulation is applied to the same shell structure shown in Fig. 1 with all laminates built using the CFRP, T300/N5208 composite system. Then, let us consider the vector of input parameter $\mathrm{p}=\left[E_{1}, E_{2}, Y, S\right]$ following a normal distribution

$N$ with mean $p_{i}$ and standard deviation $r_{i}$ represented by $p_{i} \quad N \tilde{p}_{i} ; \boldsymbol{r}_{i}$. Hn particular the statistical values of non-correlated input parameters are:

$$
\begin{aligned}
& E_{1} \sim(181.000,10.860) \quad \mathrm{GPa} \\
& E_{2} \sim N(10.300,0.618) \quad \mathrm{GPa} \\
& Y \sim N(40.000,2.400) \quad \mathrm{MPa} \\
& S \sim N(6.800,0.4080) \quad \mathrm{MPa}
\end{aligned}
$$

The formulation presented in Section 3.2 is implemented for critical Tsai number $\bar{R}$ and reliability index $b_{s}$, denoted here by $W_{m}$, and using the above mechanical properties as input parameters. To provide the fraction of the global variance $\operatorname{var}\left(W_{m}\right)$ due to each input parameter and further to calculated the respective importance measure the global Sobol first-order sensitivity index defined in Eq. (16) is used. This means that the global variance $\operatorname{var}\left(W_{m}\right)$ is explained by the contribution of partial variances as defined in Section 3.1.
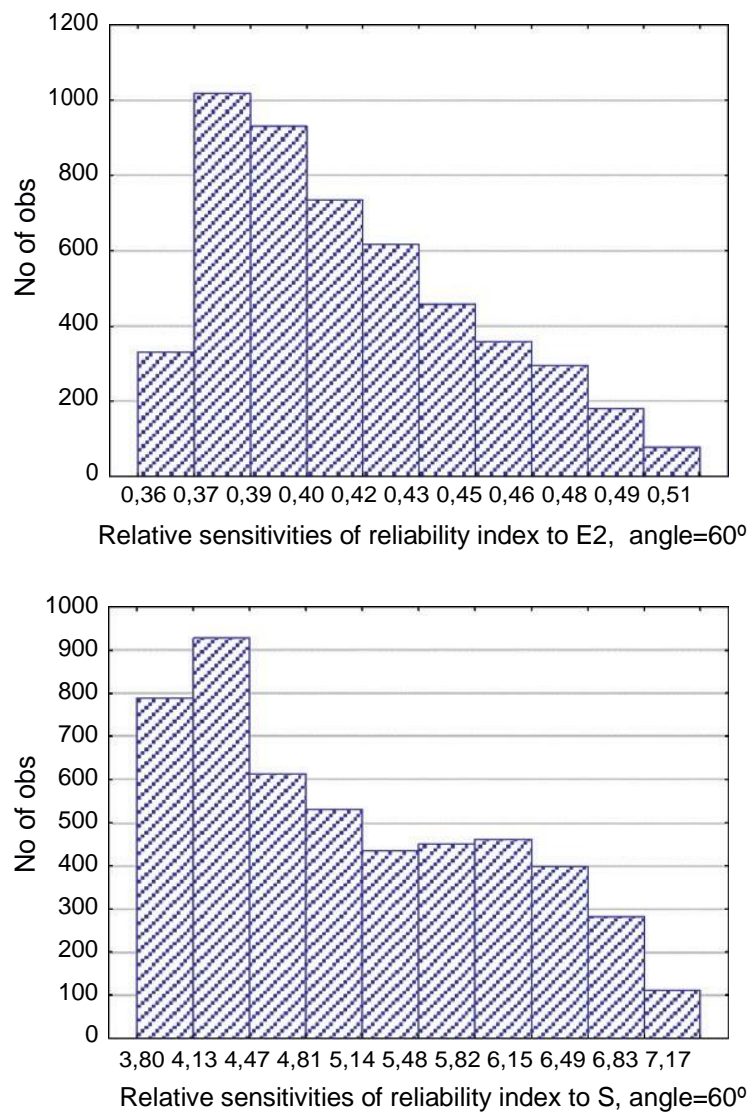
Descriptive statistics of the structural response parameters, using data from the ANN-based MCS approach for clamped composite shell.

\begin{tabular}{|c|c|c|c|c|c|c|}
\hline & $\begin{array}{l}\text { Tsai number } \\
\text { Angle }=60^{\circ}\end{array}$ & Reliability index & $\begin{array}{l}\text { Rel. sensi. of reliab. } \\
\text { index to } E_{1}\end{array}$ & $\begin{array}{l}\text { Rel. sensi. of reliab. } \\
\text { index to } E_{2}\end{array}$ & $\begin{array}{l}\text { Rel. sensi. of reliab. } \\
\text { index to } Y\end{array}$ & $\begin{array}{l}\text { Rel. sensi. of reliab. } \\
\text { index to } S\end{array}$ \\
\hline Mean & 1.154 & 2.959 & 1.671 & 0.412 & 1.852 & 5.082 \\
\hline Std. deviation & 0.022 & 0.533 & 0.349 & 0.034 & 0.281 & 0.884 \\
\hline C.V. $(\%)$ & 1.89 & 18.02 & 20.91 & 8.22 & 15.19 & 17.40 \\
\hline Minimum & 1.110 & 1.935 & 1.133 & 0.355 & 1.429 & 3.797 \\
\hline Maximum & 1.190 & 3.873 & 2.465 & 0.507 & 2.475 & 7.165 \\
\hline$N$ & 5000 & 5000 & 5000 & 5000 & 5000 & 5000 \\
\hline
\end{tabular}
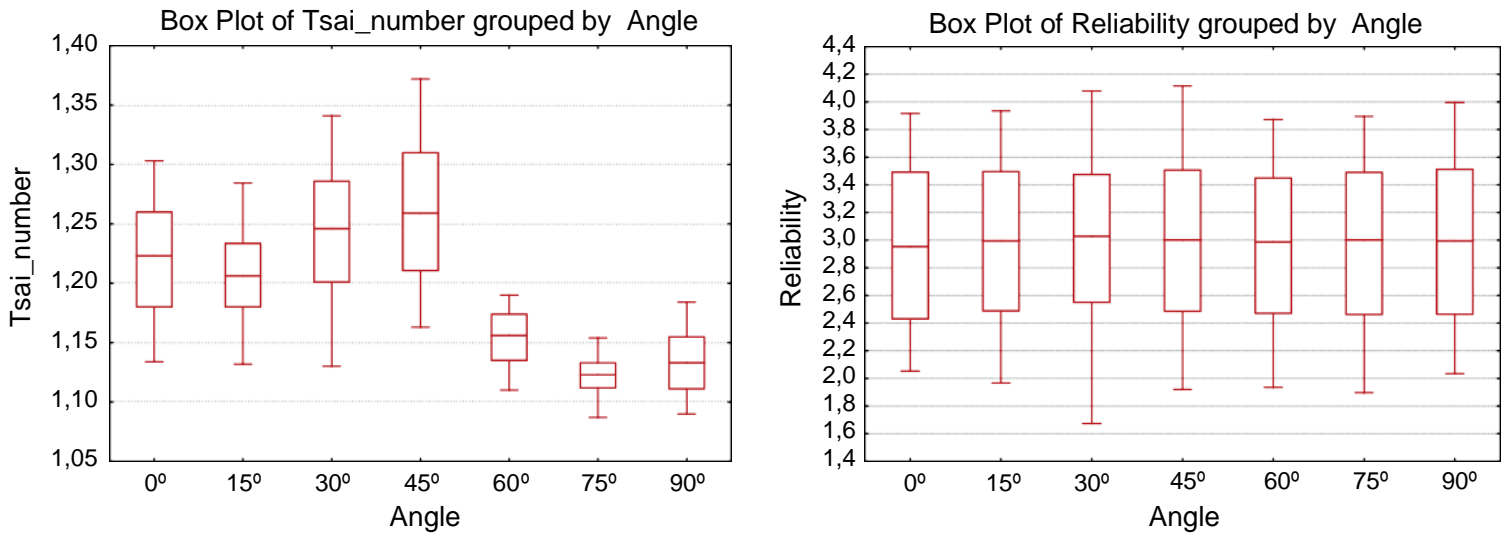

Fig. 6. Box plot of the critical Tsai number $R$, and reliability index $b_{s}$, for clamped composite shell on ply angle domain.

For the Monte Carlo simulation algorithm proposed in Section 3.2 the size samples are defined as follows:

- A set of random numbers, $N_{f}=50$, following a normal distribution $N(0,1)$ to generate the fixed values of input parameters.

- A sample matrix $\mathbf{M}_{x}$ with dimension $N_{r} \times(p-1)=100 \times 3$ to simulate the non-fixed input parameters.

A total of five thousand simulations was considered in Monte Carlo simulations $\left(N_{f} \times N_{r}\right)$ to estimate the variance of conditional expectation of structural response $\operatorname{var}\left(E\left\langle\Psi_{m} \mid \pi_{i}\right\rangle\right)$ according to Eq (13). The simulation process is implemented for each input parameter $\pi_{i}, i=1, \ldots, 4$ and the global variance $\operatorname{var}\left(\Psi_{m}\right)$ can be estimated from the twenty thousand simulations following Eq. (15).

An important aspect of the present work is to study the influence of anisotropy in the uncertainty propagation on structural response. Then, GSA is implemented as a function of ply angle, $a$. Figs, 8 and 9 show the global variance $\operatorname{var}\left(\Psi_{m}\right)$, explained by Sobol first-order sensitivity index $S_{1}$ :

$S_{l}=\frac{\operatorname{var}\left(\mathrm{E}\left(\Psi_{m} \mid \pi_{i}\right)\right)}{\operatorname{var}\left(\Psi_{m}\right)} \times 100(\%) \quad i=1, \ldots, 4$

evaluated for input parameter vector $\pi-\left[E_{1}, E_{2}, Y, S\right]$ and considering the critical Tsai number $\bar{R}$ and reliability index $\beta_{s}$ as $\Psi_{m}$ response functional respectively.

The aim of this modeling is to rank the input parameters according to variance response measure. Input parameters with higher contribution for conditional variance $\operatorname{var}\left(E\left\langle\Psi_{m} \mid \pi_{i}\right\rangle\right)$ will have higher sensitivity index $S_{i}$ taken as the global uncertainty importance measure of the input parameter $\pi$.

It is evident from Figs. 8 and 9 that the most important input parameter along ply angle domain is the transversal strength group $Y$ except for a short interval $\left[45^{\circ}, 60^{\circ}\right]$ where the shear strength $S$ are the most important.
The shear strength contributes for global variance $\operatorname{var}(\bar{R})$ along whole domain of ply angle $a$. However, this contribution does not appear for global variance $\operatorname{var}\left(\beta_{s}\right)$ except for a short interval $\left[45^{\circ}, 60^{\circ}\right]$ as previously referred.

The transversal elastic modulus $E_{2}$ has an important contribution to the global variance $\operatorname{var}(\bar{R})$ over the ply angle interval $\left[30^{\circ}, 90^{\circ}\right]$ rather than this importance computed as a fraction of the global variance $\operatorname{var}\left(\beta_{s}\right)$ is relevant only in the interval $\left[30^{\circ}, 45^{\circ}\right.$. The longitudinal elastic modulus $E_{1}$ has a marginal contribute to the global variance of both responses functional for ply angle equal to $30^{\circ}$.

An interesting comparison can be established between the results obtained from the relative sensitivities as defined in Eq. (7) and the ones obtained using the Sobol sensitivity indices. The relative sensitivities of the reliability index $\beta_{s}$ are obtained directly from ANN-MCS analysis considering 5000 simulations. Then, the mean values of relative sensitivities are obtained for each component of $\pi$ and the fractions of the contribution for the total value are calculated. This procedure is repeated for each ply angle value and the results are presented in Fig. 10. A comparison with results shown in Fig. 9 reveals that the most important differences are observed in the contribution of the longitudinal elastic modulus $E_{1}$.

\section{Aircraft wing}

Let us consider an aircraft wing-like composite panel as shown in Fig. 11. The panel thickness is equal to $0.015 \mathrm{~m}$. The structure is damped along linear side $(A B)$ and free along opposite side. One vertical load with perpendicular direction relatively to OXY plan is applied on point $C$. The structure is built by one laminate made of a carbon/epoxy composite system with mechanical properties defined in Table 1. A balanced angle-ply laminate with eight layers and stacking sequence $\left[+a /-a /+45^{\circ} /-45^{\circ}\right]$, is considered in a symmetric construction. Ply angle $a$, is referenced to the $x$-axis of 

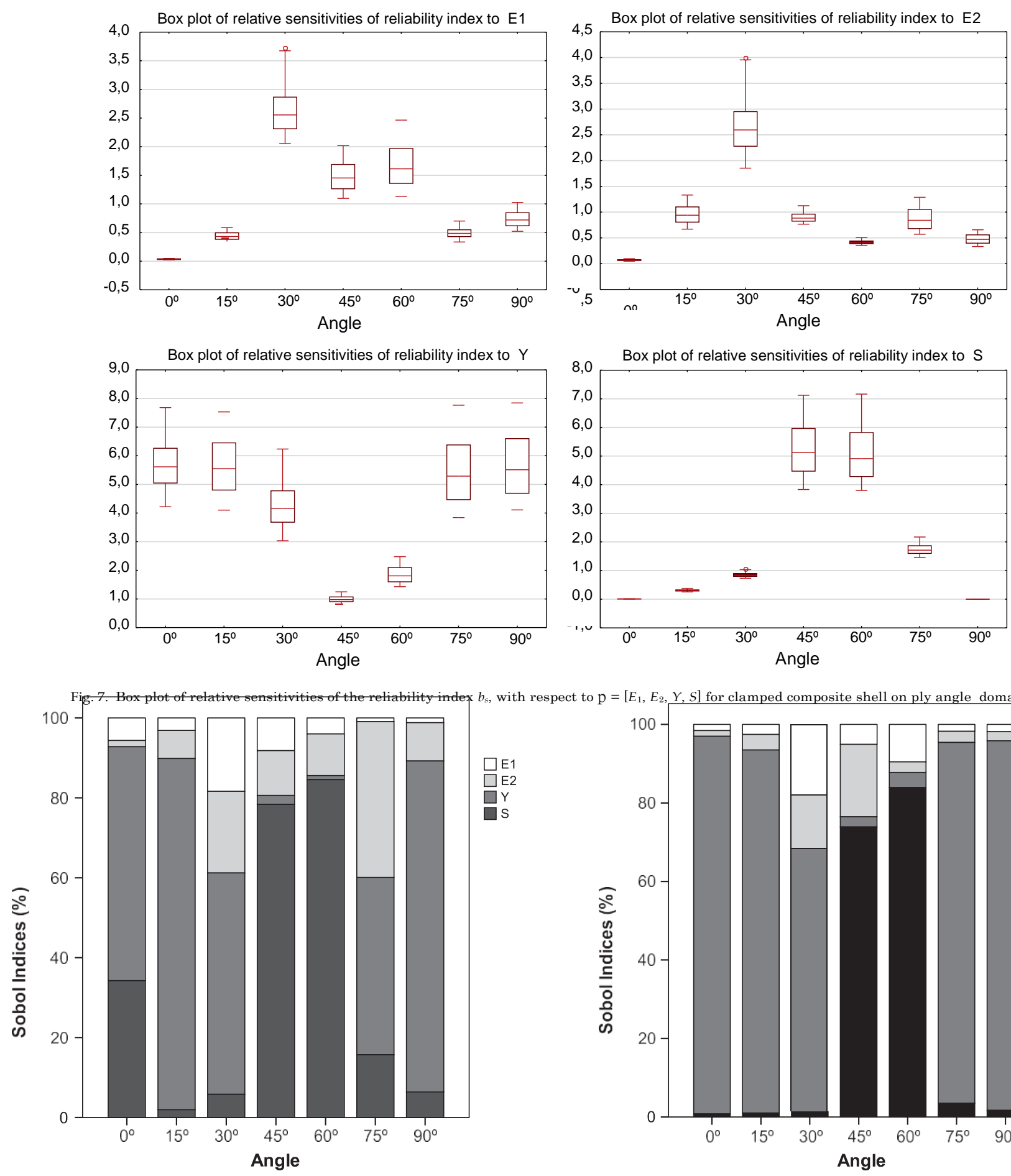

Fig. 8. Global variance varðRP, explained by Sobol first-order sensitivity index $S_{i}$ with respect to $\mathrm{p}=\left[E_{1}, E_{2}, Y, S\right]$ for clamped composite shell on ply angle domain.

the reference coordinate, as detailed in Fig. 11. All plies have same thickness.

The same shell finite element referred in previous example is used here for structural analysis. To assess reliability the previously described procedure in Eq. (2)-(6) is applied considering the vector of random variables $\mathrm{p}=\left[E_{1}, E_{2}, Y, S\right]$. The target reliability index is $b_{a}=3$ and the coefficient of variation of each random

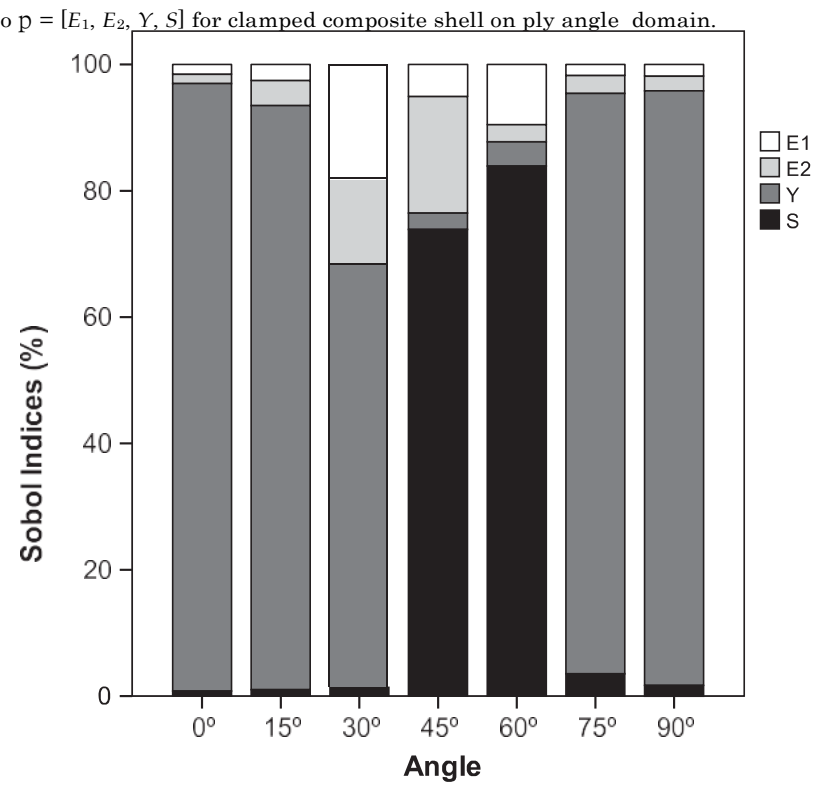

Fig. 9. Global variance var( $\left.b_{s}\right)$, explained by Sobol first-order sensitivity index $S_{i}$ with respect to $\mathrm{p}=\left[E_{1}, E_{2}, Y, S\right]$ for clamped composite shell on ply angle domain.

variable is set to $C V(p)=6 \%$, relatively to the mean value. The corresponding maximum load is plotted in Fig. 12 and it is used as the reference load for further development of the ANN supported by UDM and GA-based learning procedure.

The ANN is developed using the same UDM points defined in Table 2. After obtaining the new optimal ANN for aircraft wing-like composite panel, the uncertainty propagation analysis is per- 


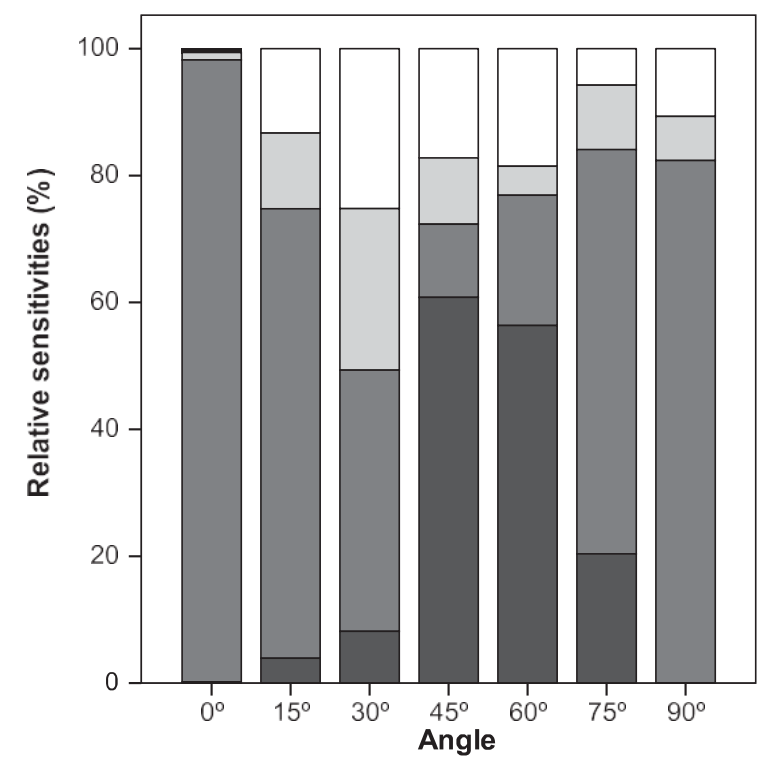

Fig. 10. Relative sensitivities of the reliability index $b_{s}$, with respect to $\mathrm{p}=\left[E_{1}, E_{2}\right.$, $Y, S]$ for clamped composite shell on ply angle domain.

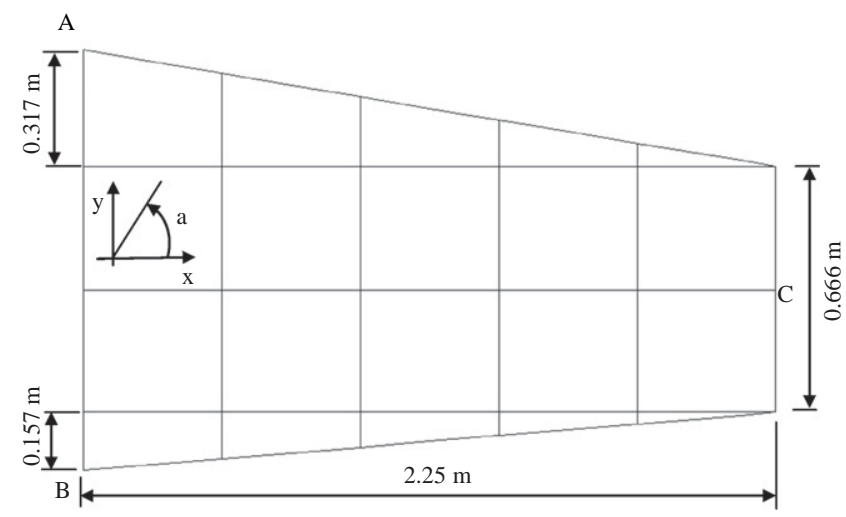

Fig. 11. Geometric definition of aircraft wing-like composite panel.

formed based on the procedure defined in Section 3.2. A set of random numbers, $N_{f}=50$, following a normal distribution $N(0,1)$ and a sample matrix $\mathrm{M}_{a}$ with dimension $N_{\mathbf{x}}(p-1)=100 \times 3$ are used in GSA algorithm for a total of twenty thousand simulations following Eq. (15). The GSA is implemented and the Sobol first-order sensitivity index $S_{i}$ is calculated as a function of ply angle, $a$. Figs. 13 and 14 show the contribution of each random variable for global variance $\operatorname{var}\left(W_{m}\right)$ using two responses functions of the composite structure. The Sobol first-order sensitivity index [11,29] is used as importance measure and the contribution is represented as a fraction of the total values at each ply angle. Fig. 13 plots the results for structural response analysis based on critical Tsai number $\bar{R}$. Similar analysis is performed using the reliability index $b_{s}$ as response functional of the structure and plotted in Fig. 14.

The most important random variable in global variance explanation of $\bar{R}$ is the transversal strength $Y$ for whole domain of ply an-

gle as shown in Fig. 13. Also the shear strength $S$ is important in interval $\left[15^{\circ}, 45^{\circ}\right]$. The longitudinal elastic modulus $E_{1}$ has relevant importance in interval $\left[45^{\circ}, 75^{\circ}\right]$ and the elastic transversal modulus $E_{2}$ is important for whole domain of ply angle $a$, except for $75^{\circ}$. Analyzing the results plotted in Fig. 14 it can be concluded that the most important random variable to explain global variance $\operatorname{var}\left(\mathrm{b}_{\mathrm{s}}\right)$ is the transversal strength $Y$ except for ply angle $a$ equal

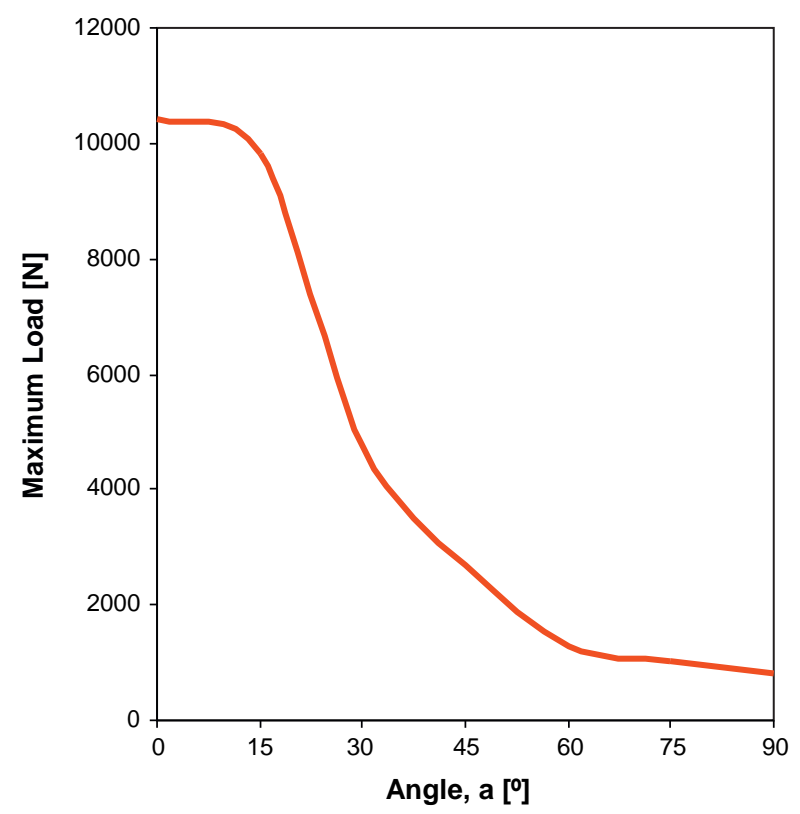

Fig. 12. Maximum load for $b_{a}=3$, solving the inverse RBDO problem for aircraft wing-like composite panel.
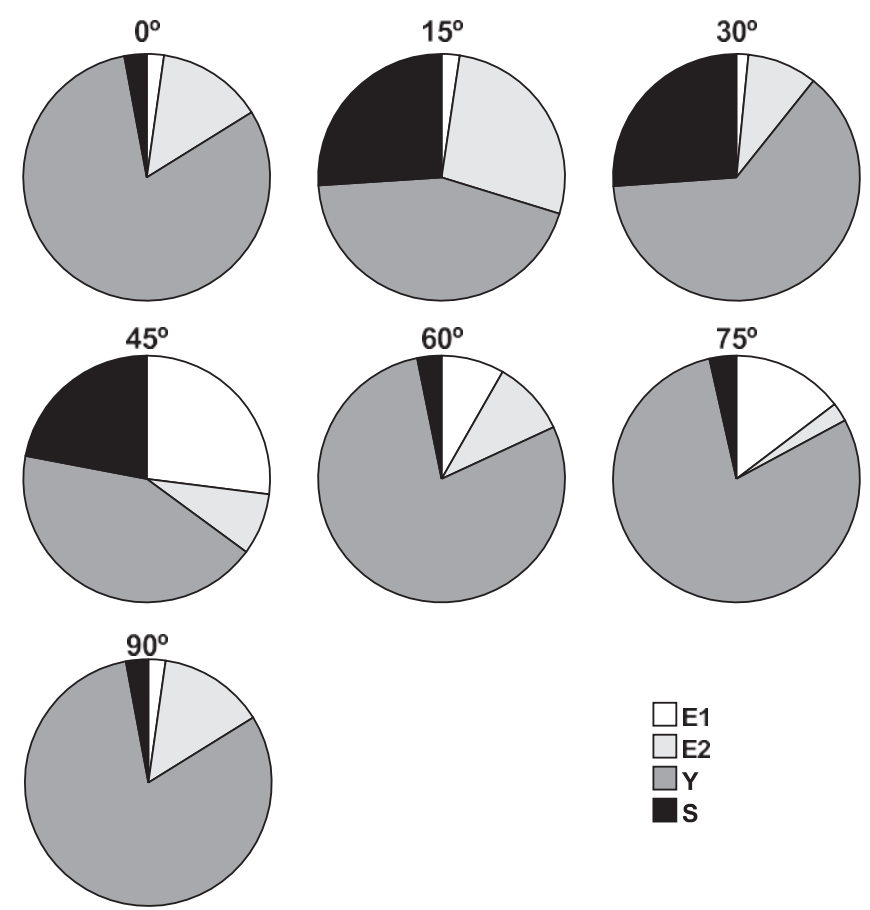

Fig. 13. Global variance varðRp, explained by Sobol first-order sensitivity index $S_{i}$ for input parameters $\mathrm{p}=\left[E_{1}, E_{2}, Y, S\right]$, aircraft wing-like composite panel.

to $30^{\circ}$ where the shear strength $S$ is the most important. Furthermore the shear strength has important contribution to explain $\operatorname{var}\left(b_{s}\right)$ in the interval $\left[15^{\circ}, 45^{\circ}\right]$. The balanced contribution of the four random variables $\mathrm{p}=\left[E_{1}, E_{2}, Y, S\right]$ for ply angle $a$ equal to $45^{\circ}$ is another relevant observation.

The global variance of critical Tsai number $\bar{R}$ and of the reliability index $b_{s}$ can be explained by Sobol indices in different manner when the ply angle $a 215^{0} ; 45^{\circ}$. Since $\bar{R}$ is associated to a deterministic analysis and $b_{s}$ is associated to a probabilistic analysis of 

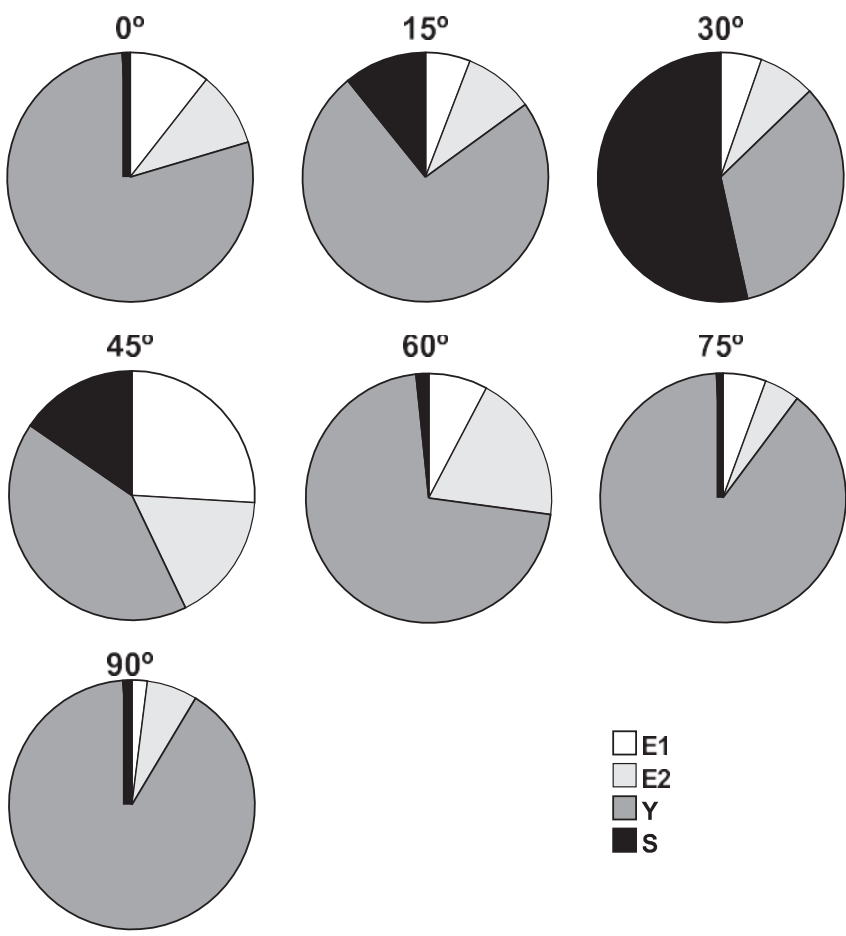

Fig. 14. Global variance $\operatorname{var}\left(b_{s}\right)$, explained by Sobol first-order sensitivity index $S_{i}$ for input parameters $\mathrm{p}=\left[E_{1}, E_{2}, Y, S\right]$, aircraft wing-like composite panel.

failure a different behavior in uncertainty propagation was expected.

\section{Conclusions}

The problem of uncertainty propagation in RBDO of composite laminate structures was studied. In particular, the effects of mechanical property deviations from the RBDO results were analyzed. The proposed ANN-based MCS approach shown that variations in the mean values of mechanical properties propagate and are even amplified in reliability index results in RBDO of composite structures. The objective of the proposed approach is to evaluate the variance of the structural response based on sensitivity indices, identifying the most important sources of uncertainty and to reduce the large number of input parameters involved in uncertainty analysis of laminated composite structures. In particular normalized indices can be established using the conditional expectation as named Sobol first-order sensitivity indices.

A study of the anisotropy influence on uncertainties propagation of composites is carried out based on the proposed methodologies. The study proves that the variability of the structural response as a function of uncertainty of the mean values can be very high. This high variability is also corroborated by evaluated relative sensitivity measures. These aspects must be considered for robust design since high structural response variability may induce a drastic reduction in the quality of the optimal design solutions for composite structures.

Based on the numerical results, the importance of measuring input parameters on structural response are established and discussed as a function of the anisotropy of composite materials. Some difference was found depending on a deterministic or a probabilistic analysis of structural failure. The uncertainty analysis propagation is very useful in designing laminated composite structures minimizing the unavoidable effects of input parameter uncertainties on structural reliability.

\section{Acknowledgment}

The authors acknowledge the financial support provided by the Foundation for Science and Technology (FCT), Portugal, through the strategic project PEst-OE/EME/UI0225/2011.

\section{References}

[1] Frangopol DM, Maute K. Life-cycle reliability-based optimisation of civil and aerospace structures. Comput Struct 2003;81(7):397-410.

[2] Rais-Rohani M, Singh MN. Comparison of global and local response surface techniques in reliability-based optimization of composite structures. Struct Multidisc Optim 2004;26:333-45.

[3] Singh BN, Yadav D, Iyengar NGR. Stability analysis of laminated cylindrical panels with uncertain material properties. Compos Struct 2001;54:17-26.

[4] Adali S, Lene F, Duvaut G, Chiaruttini V. Optimization of laminated composites subject to uncertain buckling loads. Compos Struct 2003;62:261-9.

[5] Choi JH, Lee WH, Park JJ, Youn BD. A study on robust design optimization of layered plate bonding process considering uncertainties. Struct Multidisc Optim 2008;35:531-40.

[6] Salas P, Venkataraman S. Laminate optimization incorporating analysis and model parameter uncertainties for predictable failure. Struct Multidisc Optim 2009;37:541-55.

[7] Boyer C, Béakou A, Lemaire M. Design of a composite structure to achieve a specified reliability level. Reliab Eng Syst Safety 1997;56:273-83.

[8] Singh BN, Lal A, Kumar R. Nonlinear bending response of laminated composite panels on nonlinear elastic foundation with uncertain system properties. Eng Struct 2008;30:1101-12.

[9] Carbillet S, Richard F, Boubakar L. Reliability indicator for layered composites with strongly non-linear behaviour. Compos Sci Technol 2009:69:81-7.

[10] Carrère N, Rollet Y, Retel V, Boubakar L, Maire J-F. Composites structural modelling with uncertain data. Compos Sci Technol 2009;69:60-6.

[11] Saltelli A, Ratto M, Tarantola S, Campolongo F. Sensitivity analysis practices: strategies for model-based inference. Reliab Eng Syst Safety 2006;91:1109-25.

[12] Saltelli A, Annoni P, Azzini I, Campolongo F, Ratto M, Tarantola S. Variance based sensitivity analysis of model output. Design and estimator for the total sensitivity index. Comput Phys Commun 2010;181:259-70.

[13] Tong C. Self-validated variance-based methods for sensitivity analysis of model outputs. Reliab Eng Syst Safety 2010;95:301-9.

[14] Conceição António C, Hoffbauer LN. From local to global importance measures of uncertainty propagation in composite structures. Compos Struct 2008;85:213-25.

[15] Deng J, Gu D, Li X, Yue ZQ. Structural reliability analysis for implicit performance functions using artificial neural networks. Struct Safety 2005;27:25-48.

[16] Cheng J, Li Q-S, Xiao R-C. A new artificial neural network-based response surface method for structural reliability analysis. Probab Eng Mech 2008;23:5163.

[17] Nguyen-Thien T, Tran-Cong T. Approximation of functions and their derivatives: a neural network implementation with applications. Appl Math Model 1999;23:687-704.

[18] Conceição António CA. Optimization of structures using composite materials made of polymeric matrix. PhD thesis. Portugal: Faculty of Engineering, University of Porto; 1995.

[19] Conceição António CA, Soeiro AV, Torres Marques A. Influence of physical properties randomness in laminated composites strength. In: Miravete A, editor. Proceedings of the ICCM-9, Ninth international conference on composite materials, Madrid, Spain, 12-16 July, vol. V. England: University of Zaragoza \& Woodhead Publishing Ltd.; 1993. p. 185-92. ISBN 1-85573-1401 .

[20] Conceição António CA, Torres Marques A, Gonçalves JF. Reliability based design with a degradation model of laminated composite structures. Struct Optim 1996;12:16-28.

[21] Hasofer AM, Lind NC. Exact and invariant second-moment code format. J Eng Mech Div, ASCE 1974;100:111-21.

[22] Tsai SW. Composites design. Dayton (USA): Think Composites; 1987.

[23] António CC, Hoffbauer LN. An approach for reliability-based robust design optimisation of angle-ply composites. Compos Struct 2009;90(1):53-9.

[24] Fang L, Wang Y. Number-theoretic methods in statistics. CRC Press; 1994.

[25] António CC, Hoffbauer LN. Uncertainty propagation in inverse reliability-based design of composite structures. Int J Mech Mater Des 2010;6:89-102.

[26] Cacuci DG. Sensitivity and uncertainty analysis. Theory, vol. 1. Boca Raton (FL): Chapman \& Hall/CTC Press; 2003.

[27] Conceição António CA. A hierarchical genetic algorithm for reliability based design of geometrically non-linear composite structures. Compos Struct 2001;54:37-47.

[28] Borgonovo E. A new uncertainty importance measure. Reliab Eng Syst Safety 2007;92:771-84.

[29] Sobol IM. Global sensitivity indices for nonlinear mathematical models and their Monte Carlo estimates. Math Comput Simul 2001;55:271-80.

[30] Ahmad S. Curved finite elements in the analysis of solid, shell and plate structures. PhD thesis. UK: University College of Swansea; 1969.

[31] Figueiras JA. Ultimate load analysis of anisotropic and reinforced concrete plates and shells. PhD thesis. UK: University College of Swansea; 1983. 